A. Pramesh Rao, G. Swarup and Gopal-Krishna, eds.

\title{
Theoretical Implications of Diffuse Non-Thermal Emission from Clusters of Galaxies
}

\author{
T.A. Enßlin \\ Max-Planck-Institut für Astrophysik, Karl-Schwarzschild-Str.1, \\ 85740 Garching, Germany
}

\begin{abstract}
A short review on theoretical implications of non-thermal emission (radio, extreme ultraviolet, high energy X-ray) from the intracluster medium is given. The origin of cluster radio halos and cluster radio relics is discussed within the framework of a network of processes producing a non-thermal electron population. Emphasis is given to the role of old, remnant, presently invisible relativistic plasma released by former radio galaxies.
\end{abstract}

\section{Introduction}

The space between galaxies in clusters of galaxies is filled with a hot and dilute thermal gas, which is the main component of the intra-cluster medium (ICM). Radio astronomical observations gave the first evidence for the occurrence of diffuse non-thermal phenomena in the ICM with the detection of the radio halo of the Coma cluster (Willson 1970). Recently more non-thermal signatures were detected from Coma and a number of other clusters with the observations of extreme ultra-violet (EUV) emission (Lieu et al. 1996) and high energy Xray (HEX) emission (Fusco-Femiano et al. 1999) in excess of the thermal one. The interpretation of these fluxes is still not yet settled, nor are the different classes of diffuse radio emission completely understood. We give an overview on current interpretations and theories. This should outline the physical insight we will gain from understanding the currently strongly increasing number of non-thermal detections of cluster of galaxies (Feretti 1999).

\section{Diffuse Radio Emission from Clusters of Galaxies}

Radio observations are the most sensitive probes of non-thermal processes in clusters of galaxies, and especially low frequency radio astronomy plays a crucial role since the radio spectra are steep. The diffuse radio sources can be divided into two classes: cluster radio halos and cluster radio relics ${ }^{1}$.

\footnotetext{
1 'Cluster radio relics' should not be confused with dying radio galaxies, which are also called 'radio relics'. For this reason Luigina Feretti proposed to call them 'peripheral halos', and Ron Ekers proposed 'radio flotsam'. Since all three names have disadvantages I decided to stick to the old one.
} 
The morphology of the radio halos is similar to that of the X-ray gas. They are unpolarized and occur preferentially in clusters which also show evidence for ongoing or recent cluster merger events. Cluster radio relics are irregularly shaped, more peripherally located with respect to the cluster center and sometimes linearly polarized. Both, cluster relics and halos, seem to be connected to cluster merger, but their natures are different.

\section{Cluster Radio Relics}

There is increasing evidence that the cluster relics appear at locations where shock waves of an ongoing merger event, or from the accretion flow onto the cluster are located. In the cases of Abell 2256 and Abell 1367 temperature substructures of the hot ICM gas could be detected (Briel \& Henry 1994; Donnelly et al. 1998), which support the presence of a shock wave at the location of cluster relics in these clusters. For Abell 2256, Abell 3667 and also the Coma cluster numerical simulations of merger events were satisfactorily fitted to the X-ray data, which also supports the shock wave-relic connection (Roettiger, Burns, \& Pinkney 1995; Roettiger, Burns, \& Stone 1999).

The shock wave probably accelerates electrons (and also protons) via Fermi acceleration. The accelerated electrons illuminate the cluster magnetic fields by synchrotron radiation leading to the cluster relic. The magnetic fields get compressed and therefore aligned with the shock plane, causing the radio emission to be polarized if seen edge on. Thus, the observed properties of cluster radio relics, such as as degree and direction of polarization, surface luminosity, peripheral position etc., can be understood (Enßlin et al. 1998; Roettiger, Burns, \& Stone 1999).

But shock waves are common due to the high merging fraction of cluster of galaxies, whereas cluster relics are only rarely found in clusters. This could be a surface brightness problem, or the indication of a second necessary condition (in addition to a shock wave) for the occurrence of a cluster relic.

The cluster relic $1253+275$ in Coma might tell us what this second condition is: its radio emission is morphological connected to the radio tails of the galaxy NGC 4789 (Giovannini, Feretti, \& Stanghellini 1991). Following the radio tails of that galaxy from the head to the cluster relic, one sees clear evidence for spectral steepening of the electron population in the radio spectral index, and a flattening at the location of the relic. This suggests that it is the radio plasma the galaxy releases which actually flashes up at the shock wave and not the normal inter-galactic medium (IGM). Radio plasma has the ideal composition for this: evidently magnetic fields and a pre-accelerated electron population are present. It is not necessary for a cluster radio relic, as it is the case for $1253+275$, that the source of the radio plasma is nearby or visible, since also old invisible radio plasma sitting in the IGM could be re-activated by shock waves.

\section{Radio Ghosts}

Active radio galaxies fill large volumes in the IGM with radio plasma. This plasma becomes rapidly invisible to radio telescopes due to inverse Compton (IC) and synchrotron energy losses of the relativistic electrons. Afterwards it 
might form an invisible, but possibly important phase of the IGM. Due to the difficult detectability the name 'radio ghosts' for patches of this remnant radio plasma $^{2}$ was proposed (Enßlin 1999).

After injection of the radio plasma it will expand or contract until it reaches pressure equilibrium with the surrounding medium. Its pressure is given by that of the confined relativistic particles and the magnetic fields, assumed to be in rough energy equipartition. Therefore magnetic field energy density in radio ghosts should be lower, but of the order of the thermal energy density of the environment. Subsonic turbulence in this environment, which has an energy density below the thermal energy density, is therefore not strong enough to overcome the magnetic elastic forces of the radio ghost. Sonic or supersonic turbulence, which is e.g. expected in giant merger events of cluster of galaxies, can 'shred' the ghost into smaller pieces. The size of such pieces will be comparable to the eddy size of the turbulence. Since a typical turbulent spectrum has less energy density on smaller scales there is a length-scale below which the turbulence is not able to overcome magnetic forces. Turbulent erosion of radio ghosts should stop at this length-scale, leaving small-scale patches of still unmixed old radio plasma.

Radio ghosts are practically invisible as long as their electron population remains at low energies. But if the population is re-accelerated the ghost becomes radio luminous again. This can happen when the ghost is dragged into a large-scale shock wave, e.g. in a merger event of clusters of galaxies or at the accretion shock where the matter is falling onto a cluster. The emission region is expected to be irregularly shaped, and should exhibit linear polarization due to the compression of the magnetic fields in the shock. Such regions are indeed observed at peripheral locations of a few clusters of galaxies: the cluster radio relics. Further observable signatures of radio ghosts are discussed in Enßlin (1999), Medina Tanco \& Enßlin (2000), and Enßlin \& Kaiser (2000).

\section{Cluster Radio Halos}

The origin of the relativistic electrons and magnetic fields in the ICM, which are necessary for the synchrotron process which produces the radio halo, is not known. The magnetic fields could be primordial (Olinto 1997), injected by galactic winds (Kronberg, Lesch, \& Hopp 1999; Völk \& Atoyan 1999), or by radio galaxies (Daly \& Loeb 1990; Okoye \& Onuora 1996), produced in shock waves of the large scale structure formation (Kang, Rachen, \& Biermann 1997), amplified in the turbulence from galaxy motion (Roland 1981; de Young 1992; Sánchez-Salcedo, Brandenburg, \& Shukurov 1998) and cluster formation (Roettiger, Stone, \& Burns 1999; Dolag, Bartelmann, \& Lesch 1999), or of

\footnotetext{
${ }^{2}$ More nomenclature: 'Since the term 'ghost' does not fit the context from the viewpoint of Hindu philosophy, Gopal Krishna proposed 'radio fossil', drawing upon the Indian mythological tale of lady Ahilya (ref: the epic Ramayana, circa 2000 B.C.). Turned into a fossil while meditating, Ahilya was revived to life at the touch of Lord Rama's feet, as the Lord passed her way on a pre-ordained track during his 14-year exile in the forest. Likewise, 'radio fossils' can spring back to a radiant life upon passage of a shock wave.
} 


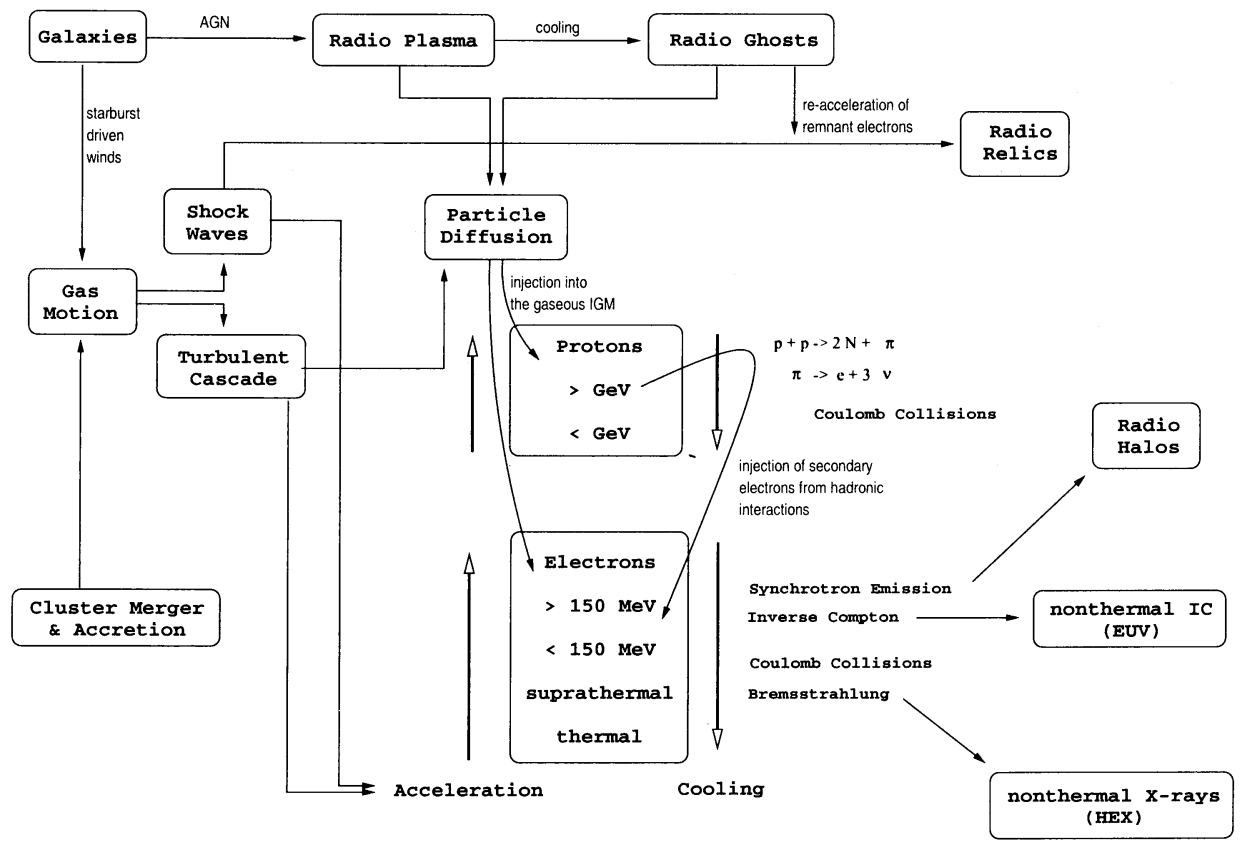

Figure 1. Network of processes possibly responsible for non-thermal cluster emission. Description in the text.

another unknown origin. Harris \& Miley (1978) and Komissarov (1985) proposed that radio halos are superpositions of large numbers of relic radio galaxis.

There are also many possible sources for the relativistic electrons. Fig. 1 gives an overview about the current theories and their dependencies. In the following the different parts of the diagram are explained and references to the literature are given:

The energy source of all non-thermal processes in clusters should be either the kinetic energy of matter falling onto clusters, or the outflows from galaxies. The latter can be divided in galactic winds, which are strongest for starburst galaxies, and ejection of radio plasma from an AGN. All these processes can produce shock waves and inject turbulence into the ICM, and therefore produce conditions where Fermi mechanisms accelerate particles (Jaffe 1977; Schlickeiser, Sievers, \& Thiemann 1987; Roettiger, Burns, \& Loken 1993; Tribble 1993; Völk, Aharonian, \& Breitschwerdt 1996; Deiss et al. 1997; Eilek \& Wetherall 1999; Wetherall \& Eilek 1999).

The non-thermal particles could be accelerated out of the thermal pool (Liang 1999), or they were produced by radio galaxies (Jaffe 1977; Rephaeli $1977 ; 1979$; Valtaoja 1984). In the latter case, they had to escape out of the radio plasma, which is difficult since the necessary diffusion across magnetic fields is a extremely slow process. Turbulence in the ICM can strongly increase that diffusion coefficient, and especially shortly after mayor merger events there exists a short time window when particles might be able to escape (Enßlin 1999). 
The non-thermal electron population produces bremsstrahlung, inverse Compton and synchrotron emission, which allows us to detect its presence, but also leads to energy losses which have to be compensated by re-acceleration (Jaffe 1977; Giovannini et al. 1993; Brunetti et al. 1999) or injection of fresh electrons. If the radio electrons are injected and not accelerated in-situ, the sources have to be distributed throughout the cluster volume. Otherwise, if only a few located sources produce the electrons, cooling times of the electrons are much shorter than plausible travel times from the source to the boundaries of the observed radio halos (Jaffe 1977; but see Holman, Ionson, \& Scott 1979; Ragot \& Kirk 1997). A promising injection mechanism is therefore secondary particle production from hadronic interactions of relativistic protons with the background gas (Dennison 1980; Vestrand 1982; Blasi \& Colafrancesco 1999):

$$
\begin{aligned}
p+p & \rightarrow 2 N+\pi^{ \pm} \\
\pi^{ \pm} & \rightarrow \mu^{ \pm}+\nu_{\mu} / \bar{\nu}_{\mu} \rightarrow e^{ \pm}+\nu_{e} / \bar{\nu}_{e}+\nu_{\mu}+\bar{\nu}_{\mu}
\end{aligned}
$$

The lifetime of relativistic protons in the ICM is of the order of the Hubble time, or larger (Berezinsky, Blasi, \& Ptuskin 1997). Thus they are able to travel large distances from their sources before they release their energy. The production of electrons via charged pions has to be accompanied by gamma ray production via neutral pions (Vestrand 1982; Enßlin et al. 1997; Colafrancesco \& Blasi 1998; Blasi 1999):

$$
\begin{aligned}
p+p & \rightarrow 2 N+\pi^{o} \\
\pi^{o} & \rightarrow 2 \gamma
\end{aligned}
$$

Thus clusters with radio halos might have gamma-ray halos, which would be, if detected, a direct proof for a hadronic origin of radio halos.

\section{The Electron Spectrum}

The electron spectrum is formed by various injection, cooling and acceleration processes, which should leave their fingerprints on it. Fig. 2 shows a compilation of the electron spectrum in the Coma cluster. Note, that the EUV and the HEX upper limits actually result from detections of excess emissions (compared to the thermal X-ray emission at these energies; Lieu et al. 1996; Fusco-Femiano et al. 1999), so that some of the upper limits actually could be data points.

The EUV-excess could result from a low temperature gas (Lieu et al. 1996). But this is problematic, since one would expect the presence of resonance lines, which are not detected (Dixon, Hurwitz, \& Ferguson 1996). Therefore an IC origin of the excess is very likely. The scattered photon field could be the starlight of the galaxies, which could explain the narrow radial profile of the emission, but this would require a very high relativistic electron energy density in Coma (Enßlin, Lieu, \& Biermann 1999). Probably the excess is due to IC scattered cosmic microwave background (CMB) photons (Hwang 1997; Enßlin \& Biermann 1998; Bowyer \& Berghöfer 1998), which would require a strong electron population around $150 \mathrm{MeV}$, not unreasonable since cooling is slowest at this energy (Sarazin \& Lieu 1998). In order to connect this data point with the radio electrons a huge step in the electron spectrum is required, if the Faraday 


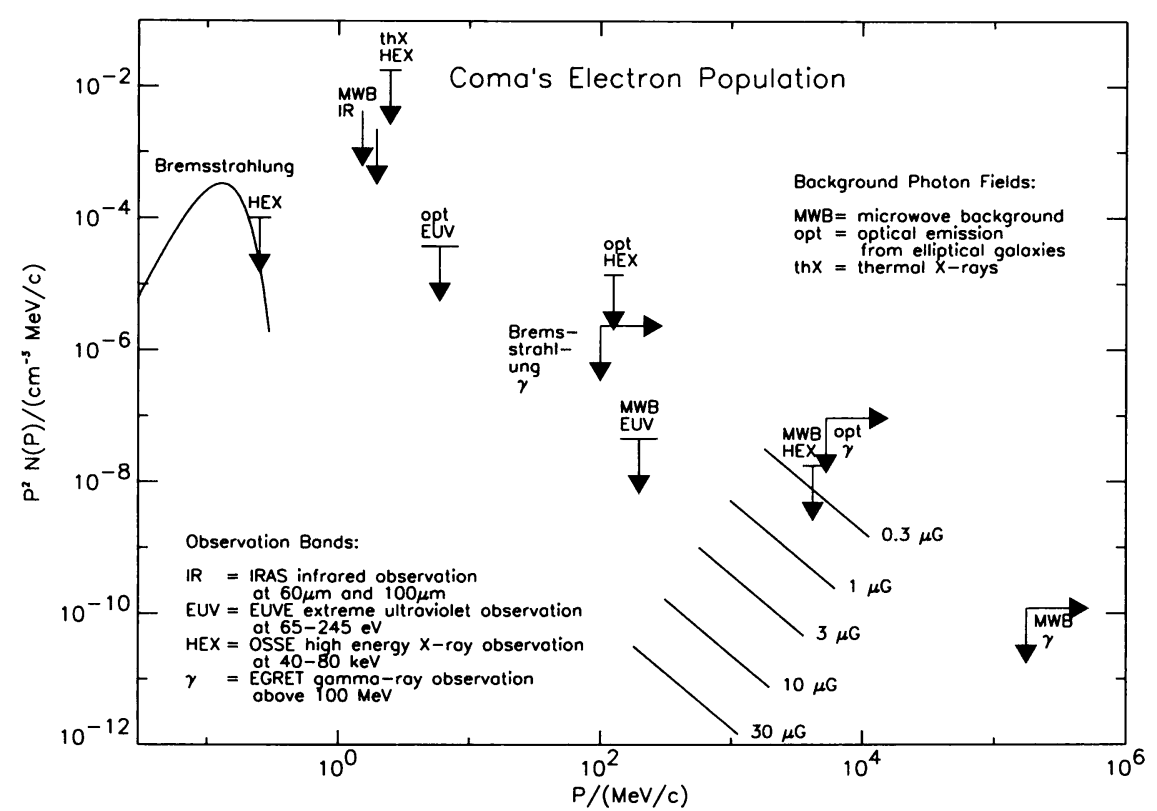

Figure 2. The electron spectrum in the center of the Coma cluster (from Enßlin \& Biermann 1998). The solid line below $1 \mathrm{MeV} / c$ is the thermal electron spectrum and the lines around $1 \mathrm{GeV} / c$ give the radio emitting electrons for several magnetic field strength. The upper limits result from several upper limits to possible IC and bremsstrahlung processes.

rotation determined magnetic field strength of $6 \mu \mathrm{G}$ (Feretti et al. 1995) in Coma is correct. An electron population which was accelerated e.g. in a violent merger event in the past would have produced such a step due to cooling (Enßlin et al. 1999; Sarazin 1999; Atoyan \& Völk 1999). If the magnetic fields are inhomogeneous, the required step can be further reduced, since cooling produces a electron distribution spatially anti-correlated with the magnetic fields, thus hiding the EUV producing electrons from radio observations.

The HEX-excess can not result from IC scattered starlight photons, since this would violate other limits to the electron spectrum (see Fig. 2). In principle it could result from IC scattered CMB photons, but this would require magnetic field strength of $0.16 \mu \mathrm{G}$ since otherwise the synchrotron emission of the responsible electrons would exceed the observed radio halo of Coma (Fusco-Femiano et al. 1999). This is in strong contradiction to Faraday rotation measurements. Therefore it is very likely, that a supra-thermal electron population produces the HEX excess by bremsstrahlung (Enßlin et al. 1999; Sarazin \& Kempner 1999). Such a population is expected due to turbulent particle acceleration of thermal electrons (Dogiel 1999; Liang 1999; Blasi 2000), and it can be confirmed with future CMB telescopes due to its non-thermal IC signature (Enßlin \& Kaiser 2000). 
Acknowledgments. I like to thank the scientific and the local organizing committee for the invitation to this stimulating conference. I am grateful for financial travel support from the International Astronomical Union (IAU) and the Deutsche Forschungsgemeinschaft (DFG). During this journey I enjoyed the friendly hospitality at the National Center for Radio Astrophysics (NCRA) in Pune, at the Inter University Centre for Astronomy and Astrophysics (IUCAA) in Pune, and the Raman Research Institute (RRI) in Bangalore.

\section{References}

Atoyan, A. M., \& Völk, H. J., 1999, ApJ, in press, astro-ph/9912557

Berezinsky, V. S., Blasi, P., \& Ptuskin, V. S. 1997, ApJ, 487, 529

Blasi, P., \& Colafrancesco, S. 1999, Astroparticle Physics, 12, 169

Blasi, P. 1999, ApJ, 525, 603

Blasi, P. 2000, ApJ, in press, astro-ph/0001344

Bowyer, S., \& Berghöfer, T. W. 1998, ApJ 506, 502

Briel, U.G., \& Henry, J.P. 1994, Nature, 372, 439

Brunetti, G., Setti, G., Feretti, L., \& Giovannini G. 1999, submitted to MNRAS

Colafrancesco, S., \& Blasi, P. 1998, Astroparticle Physics, 9, 227

Daly, R.A., \& Loeb, A. 1990, ApJ, 364, 451

Deiss, B. M., Reich, W., Lesch, H. \& Wielebinski, R. 1997, A\&A, 321, 55

Dennison, B. 1980, ApJ, 239, 93

de Young, D. S. 1992, ApJ, 386, 464

Dixon, W. V., Hurwitz, M., \& Ferguson, H. C. 1996, ApJ 469, L77

Dolag, K., Bartelmann, M., \& Lesch, H. 1999, A\&A, 348, 351

Donnelly, R. H., Markevitch, M., Forman, W., Jones, C., David, L. P., Churazov, E., \& Gilfanov, M. 1998, ApJ, 500, 138

Jaffe, W. J. 1977, ApJ, 212, 1

Eilek, J., \& Wetherall, J. C. 1999, in Ringberg ${ }^{3}, 249$

Enßlin, T. A., Biermann, P. L., Kronberg, P. P., \& Wu, X.-P. 1997, ApJ, 477, 560

Enßlin, T. A., \& Biermann, P. L. 1998, A\&A, 330, 90

Enßlin, T. A., Biermann, P. L., Klein, U., \& Kohle, S. 1998, A\&A, 332, 39

Enßlin, T. A., Lieu, R., \& Biermann, P. L. 1999, A\&A, 344, 409

Enßlin, T. A. 1999, in Ringberg ${ }^{3}$, 275, astro-ph/9906212

Enßlin, T. A., \& Kaiser, C. R. 2000, in preparation

Feretti, L., Dallacasa, D., Giovannini, G., \& Tagliani, A. 1995, A\&A, 302, 680

Feretti, L. 1999, these proceedings

${ }^{3}$ Ringberg Workshop on 'Diffuse Thermal and Relativistic Plasma in Galaxy Clusters', Eds: H.

Böhringer, L. Feretti, P. Schuecker, MPE Report No. 271, 1999 
Fusco-Femiano, R., dal Fiume, D., Feretti, L., Giovannini, G., Grandi, P., Matt, G., Molendi, S., \& Santangelo, A. 1999, ApJ, 513, L21

Giovannini, G., Feretti, L., \& Stanghellini, C. 1991, A\&A, 252, 528

Giovannini, G., Feretti, L., Venturi, T., Kim, K. -T. \& Kronberg, P. P. 1993, ApJ, 406, 399

Harris, D. E., \& Miley, G. K. 1978, A\&AS, 34, 117

Holman, G. D., Ionson, J. A. \& Scott, J. S. 1979, ApJ, 228, 576

Hwang, C.-Y. 1997, Science 278, 1917

Kang, H., Rachen, J. P., \& Biermann, P. L. 1997, MNRAS, 286, 257

Komissarov, S. S. 1985, Soviet Astronomy, 29, 382

Kronberg, P. P., Lesch, H., Hopp, U. 1999, ApJ 511, 56

Liang, H. 1999, in Ringberg ${ }^{3}, 33$

Lieu, R., Mittaz, J. P. D., Bowyer, S., Breen, J. O., Lockman, F. J., Murphy, E. M., \& Hwang, C. -Y. 1996, Science, 274, 1335

Medina-Tanco, G., Enßlin, T. A., 2000, in preparation

Okoye, S. E. \& Onuora, L. I. 1996, MNRAS, 283, 1047

Olinto, V.A., 1997, in 3rd RESCEU International Symposium on "Particle Cosmology", University of Tokyo, 1997, astro-ph/9807051

Ragot, B. R., \& Kirk, J. G. 1997, A\&A, 327, 432

Rephaeli, Y. 1977, ApJ, 212, 608

Rephaeli, Y. 1979, ApJ, 227, 364

Roettiger, K., Burns, J., \& Loken, C. 1993, ApJ, 407, L53

Roettiger K., Burns, J. O., Pinkney J. 1995, ApJ, 453, 634

Roettiger, K., Stone, J. M., \& Burns, J. O. 1999, ApJ, 518, 594

Roettiger, K., Burns, J. O., \& Stone, J. M. 1999, ApJ, 518, 603

Roland, J. 1981, A\&A, 93, 407

Sánchez-Salcedo, F. J., Brandenburg, A., \& Shukurov, A. 1998, Ap\&SS, 263, 87

Sarazin, C. L., \& Lieu, R. 1998, ApJ, 494, L177

Sarazin, C. L. 1999, ApJ, 520, 529

Sarazin, C. L., \& Kempner, J. C. 1999, ApJ, in press, astro-ph/9911335

Schlickeiser, R., Sievers, A., \& Thiemann, H. 1987, A\&A, 182, 21

Tribble, P. C. 1993, MNRAS, 263, 31

Valtaoja, E. 1984, A\&A, 135, 141

Vestrand, W. T. 1982, AJ, 87, 1266

Völk, H. J., Aharonian, F. A., \& Breitschwerdt, D. 1996, Space Sci. Rev., 75, 279

Völk, H. J., \& Atoyan, A.M. 1999, in Ringberg ${ }^{3}, 99$

Wetherall, J.C., \& Eilek, J. 1999, in Ringberg ${ }^{3}, 255$

Willson, M. A. G. 1970, MNRAS, 151, 1 